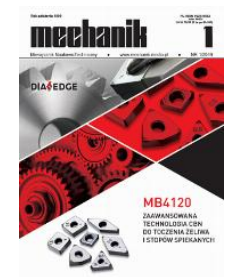

How to cite this article:

Authors: Michał Karpiuk, Maciej Malik, Magdalena Przytocka, Katarzyna Czajkowska-Sabat, Witold Sujka

Title of article: „Computer aided manufacturing of compression garments used for rehabilitation of burn and post-

operative scars"

Mechanik, No. 1 (2019)

DOI: https://doi.org/10.17814/mechanik.2019.1.11

\title{
Computer aided manufacturing of compression garments used for rehabilitation of burn and post-operative scars
}

\author{
MICHAŁ KARPIUK \\ MACIEJ MALIK \\ MAGDALENA PRZYTOCKA \\ KATARZYNA CZAJKOWSKA-SABAT \\ WITOLD SUJKA*
}

\author{
Dr inż. Michał Karpiuk, karpiuk@mech.pk.edu.pl, https://orcid.org/0000-0002-1406-4502 - Wydział Mechaniczny Politechniki \\ Krakowskiej, Kraków, Polska \\ Maciej Malik, Magdalena Przytocka - Koło Naukowe Inżynierii Produkcji, Wydział Mechaniczny Politechniki Krakowskiej, Kraków, Polska \\ Mgr inż. Katarzyna Czajkowska-Sabat, katarzyna.sabat@tricomed.com - Tricomed S.A., Łódź, Polska \\ Dr inż. Witold Sujka, witold.sujka@tricomed.com, https://orcid.org/0000-0003-0293-6681 - Tricomed S.A., Łódź, Polska
}

The article describes a computer-aided manufacturing of compression garments used for rehabilitation of burn and post-operative scars implemented in Tricomed S.A company. Steps of manufacturing processes include 3D scanning, obtaining control parameters for a self-generating CAD templates from STL file, conversion control parameters depending on the degree of compression and type of knitwear, generating DXF files for the cutting machine, sewing.

KEYWORDS: CAD, CAM, 3D scanning, reverse engineering, compression therapy, non-contact measuring

\section{Introduction}

Increasing competition requires companies to minimize costs, maximize efficiency, and offer improved, personalized products and services. The method of increasing the competitiveness is, among others, improving the manufacturing process in such a way as to obtain the most innovative products after using the latest available technology. It is also important to implement customer facilities, such as the ability to collect patient parameters remotely and to reduce the waiting time to a minimum.

Tricomed S.A. modernized the technology of producing compression garments for the treatment of burn and post-operative scars, and introduced CODOPRESS ${ }^{\circledR}$ Premium products. The traditional production of this type of product consisted of stapling fabric knitwear, which each time involved drawing paper patterns based on manual measurements (using a measuring tape) at the company's headquarters. This conventional measuring method used in tailoring is time consuming and not very accurate, and also requires direct contact with the patient's body, which may cause discomfort to him. The use of innovative reconstructive engineering techniques in conjunction with the capabilities of the 3D CAD system enables the non-contact and noninvasive measurements of individual parts of the patient's body, and automates the pattern preparation stage.

In this paper, these possibilities are presented on the example of a compression glove.

Tricomed S.A., in cooperation with the Cracow University of Technology and the Łódź University of Technology, developed and implemented computer-aided technology for the production of a whole range of compression products, including armbands, lower leg or thigh bands, as well as socks, knee socks, stockings, reforms and t-shirts. 


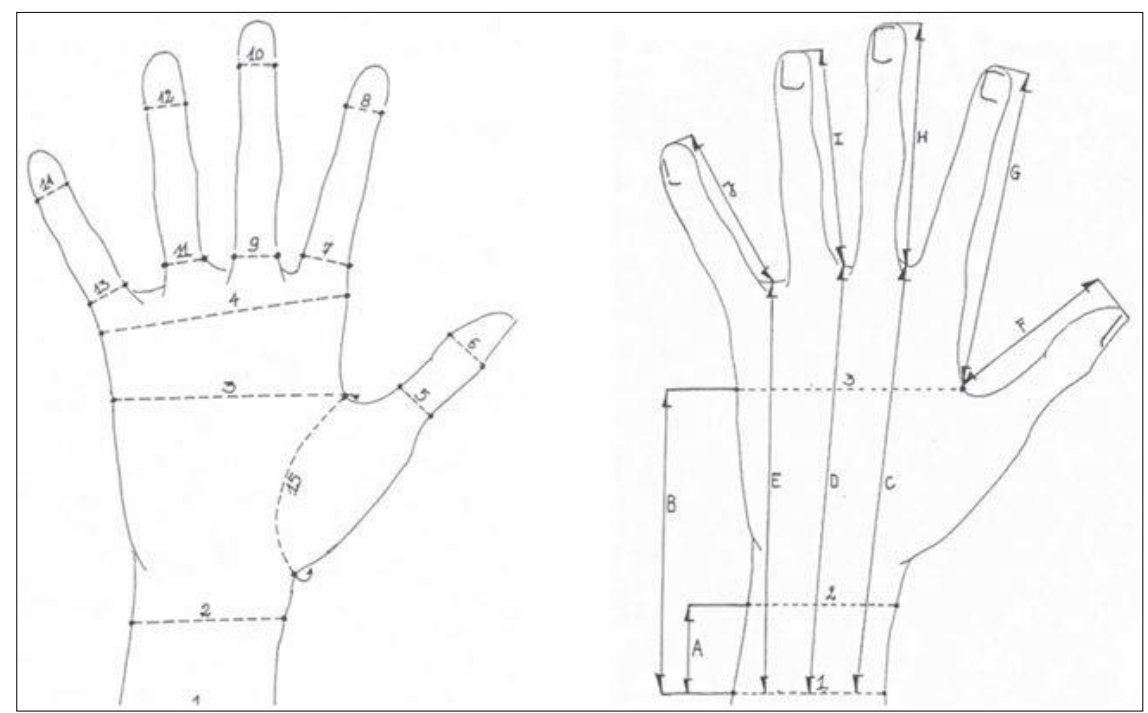

Fig. 1. Fragment of the CODOPRESS ${ }^{\circledR}$ glove measuring report

\section{Manufacture of compression products for rehabilitation}

Production of compression garments intended for rehabilitation is discussed on the example of the CODOPRESS ${ }^{\circledR}$ Premium glove. It is made of four elements. The base of the glove is a pattern reflecting the outer and inner part of the hand. After folding along the axis of symmetry, the pattern is sewn together with three inserts between the fingers: small and ring, ring and middle, as well as middle and index.

Each type of band (including gloves) is assigned a measurement protocol, containing dimensions necessary to prepare patterns. The places and names of the measurements are presented in fig. 1 . The circumferences are marked with numbers from 1 to 15 , and the lengths with letters from A to J.

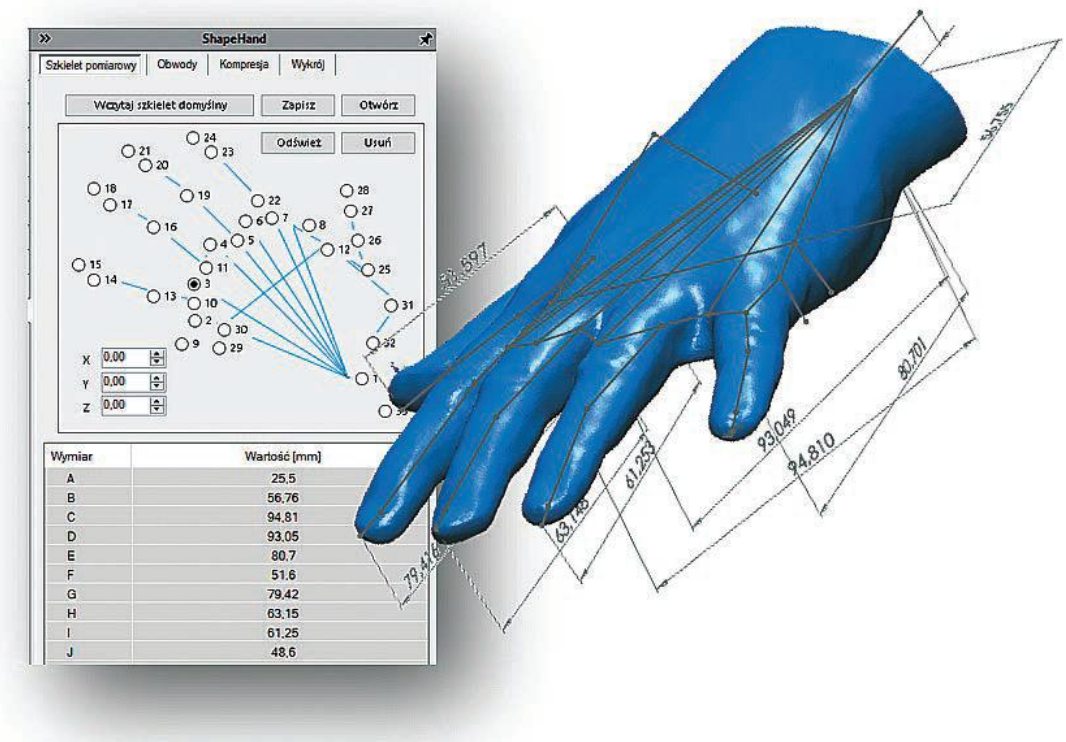

Fig. 2. "Measurement skeleton" tab of the ShapeHand add-on

Technology used so far for the production of compression bands was based on obtaining a pattern by hand cutting, based on a previously drawn paper template. In order to automate this stage of the production process, the paper prototype was replaced by an auto-generating CAD template in the form of an SLDPRT (SolidWorks Part) file. It contains the outline of the pattern in the form of a sketch - dimensioned and equipped with features enabling the model to be rebuilt together with the change of control parameters (circumference and length). Full parameterization of dimensions in the CAD template was possible due to the use of dependencies based on dimensions, global variables, file properties, mathematical functions, rules and results of other dependencies. 


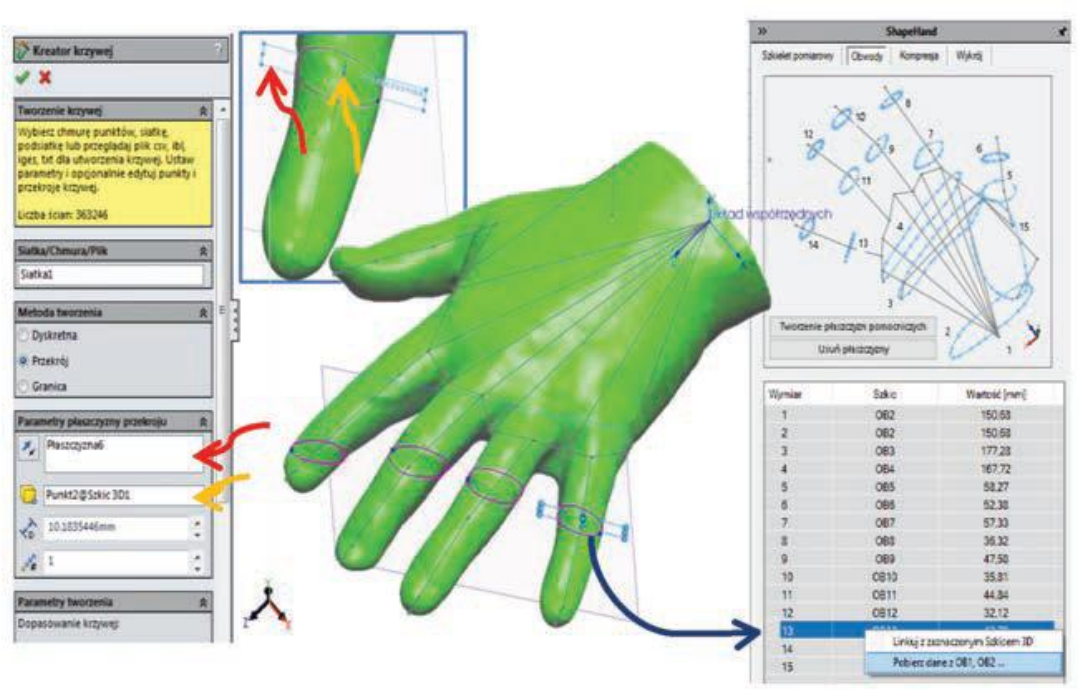

Fig. 3. "Measurement skeleton" tab of the ShapeHand add-on

Collection of the patient's dimensional data necessary to generate the correct pattern from the autogenerating template takes place in the 3D CAD system. The conventional measuring method has been replaced by a non-contact method - 3D scanning. The process of computer-aided manufacturing process begins at the stage of first contact with the patient.

The point cloud, which is the result of 3D scanning, is subject to appropriate editing to eliminate any errors. There is an automatic recognition and filling of defects in places not covered by the measurement, removal of unnecessary fragments of the mesh and its smoothing. The mesh prepared in this way is loaded into the SOLIDWORKS program equipped with the ScanTo3D add-in integrated with it.

Then the default measuring frame in the form of a 3D sketch is loaded into the file with the imported mesh (fig. 2). The user must fit it to the 3D model. Functionality discussed was achieved thanks to the ShapeHand add-on, which was specially designed and programmed for Tricomed S.A.

The ShapeHand add-on also allows to update, delete or save the current skeleton to a file. Length values (marked with letters from A to J) are taken from the frame thus modified, which are later used to rebuild the CAD pattern template.

The subsequent stage of data acquisition, allowing for the reconstruction of the CAD pattern template, is the measurement of circumferences in places indicated in the measurement report. In the case of a compression glove, these are the circumferences of the fingers and hands.

In this case, it is necessary to generate curves using the "Curve Wizard", which is available in the ScanTo3D add-on, and measure their length (OB1 ... OB15) (fig. 3).

Due to the lack of access to the API, this process could not be automated in this area. However, the ScanTo3D add-on allows to generate a spline type curve by setting "Cross Section" as the creation method and selecting the appropriate plane along with the point belonging to the measuring skeleton. "Curve creator" generates curves that intersect the hand model (grid) in the selected plane on one 3D sketch.

The system user must leave one circumference in the 3D sketch, dimension it, and then download it to ShapeHand. The length and circumference values taken in this way must be recalculated taking into account the compression ratio and the type of knitted fabric chosen. For this purpose, the ShapeHand tab has been prepared with the "Compression" tab. Materials have also been implemented, to which new characteristics can be added by entering their names and coefficients characterizing the material.

To calculate the compression, a method based on Laplace's law was used, which describes the relationship between unit pressure exerted on a cylindrical model and circumferential force in a fabric strip [1]:

$$
P=\frac{2 \pi F}{G_{1} S}
$$

where: $P[\mathrm{hPa}]$ - unit pressure, $F[\mathrm{cN}]$ - circumferential force in the material strip width $s, G_{1}[\mathrm{~cm}]$ - model circumference at a specific place, $s[\mathrm{~cm}]$ - material strip width. 


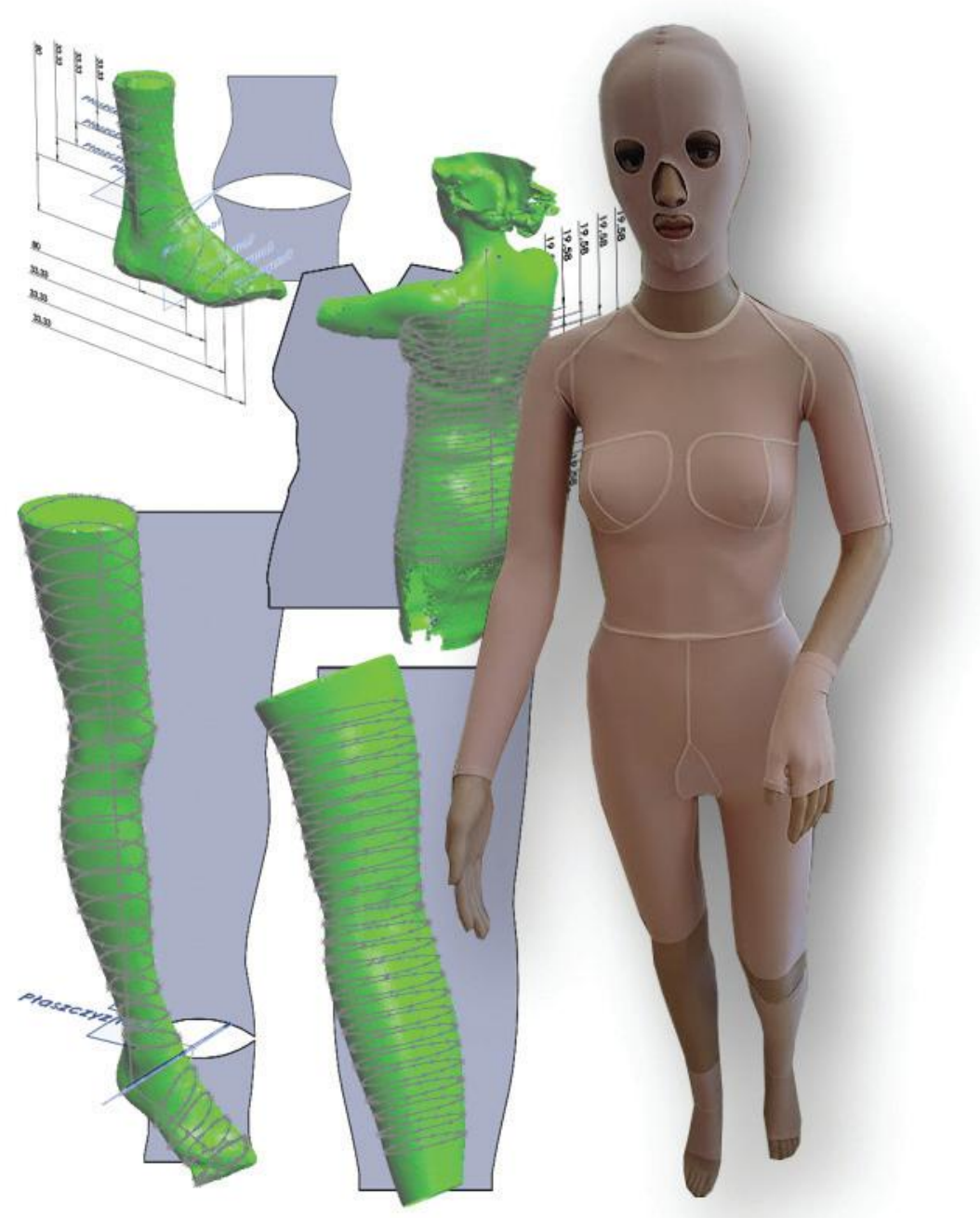

Fig. 4. Measurement of 3D models and auto-generating CAD templates of selected CODOPRESS ${ }^{\circledR}$ Premium products

Based on this relationship and information about the material used to produce compression garments, the difference between the measured circumference and the circumference after compression is calculated:

$$
G_{0}=\frac{(2 \pi a)^{\frac{1}{b}}}{\left(P G_{1} s\right)^{\frac{1}{b}}+(2 \pi a)^{\frac{1}{b}}} G_{1}
$$

where: $a, b$ - coefficients characterizing a given material, $s[\mathrm{~cm}]$ - width of the material strip, $G_{1}[\mathrm{~cm}]$ - measured circumference of the body part, $G_{0}[\mathrm{~cm}]$ - circumference of the model after compression, $P[\mathrm{hPa}]$ - unit pressure.

The subsequent phase of the technological process of manufacturing the compression band is to generate DXF files based on the prepared CAD template of the pattern and upload it to the cutting device. This stage of production has been fully automated. Therefore, just call the procedure for generating a DXF file by clicking one button of the ShapeHand application to:

- the appropriate variant of the auto-generating CAD template has been launched,

- the CAD template was supplemented with control parameters and rebuilt based on them,

- in the appropriate location, visible to the cutting machine, all the DXF files of the pattern were generated, which were included in the set of the selected compression band. 


\section{Summary}

In the case of compression bands, used during the rehabilitation of burn and post-operative scars, the use of a 3D scanner allows obtaining very accurate measurements of body circumferences. The non-contact measurement method, which is 3D scanning, allows to avoid unwanted interference in the body of the measured patient and eliminate errors that may result from the technique of manual measurement removal. The use of 3D scanning to quickly collect the necessary data has forced the development and implementation of a special scanning procedure in the company. The patient must be positioned in such a position and orientation relative to the scanner that the measured part of the body is stabilized and the hard-to-reach places are visible to the scanner [2].

The product obtained as a result of the improved technological process is immediately profiled, so it does not have to be adapted to the patient several times at later stages. The product is personalized and dimensionally suits the individual patient, so the pressure distribution of the band on the desired body surfaces is even. This was achieved, among others, due to the application of increased density of measurements performed on the 3D model in relation to the density adopted in the measurement protocols (fig. 4).

The band skeleton model can be freely modified by changing the appropriate values. If there is a place on the body where pressure is inadvisable, one can manually enlarge the circumference corresponding to this place (using the software). The dimensions obtained in this way can be transferred to the pattern template. After generating the file in DXF format, the template can be cut according to the loaded path on machines specially adapted to the knitwear.

\section{REFERENCES}

[1] Ilska A., Kowalski K., Kłonowska M., Kowalski T.M., Sujka W. "Issues regarding the design of compression products for small body circumferences". FIBRES \& TEXTILES in Eastern Europe 2016. 24, 6(120) (2016): 116-120 , https://doi.org/10.5604/12303666.1221745.

[2] Kowalski K., Karbowski K., Kłonowska M., Ilska A., Sujka W., Tyczyńska M., Włodarczyk B., Kowalski T.M. "Influence of a compression garment on average and local changes in unit pressure". FIBRES \& TEXTILES in Eastern Europe 2017. 25, 6(126): 68-74, https://doi.org/10.5604/01.3001.0010.5374. 